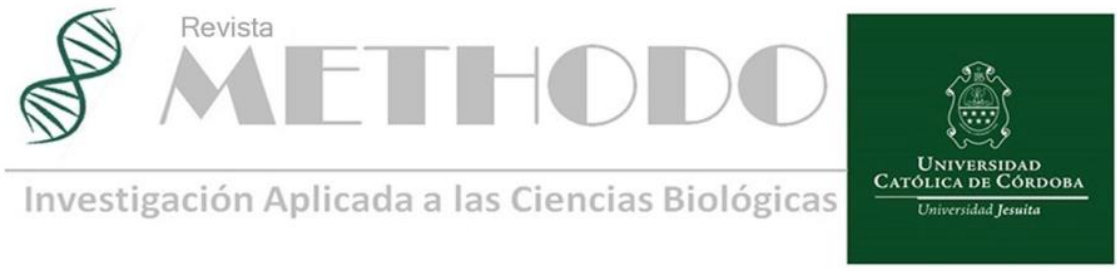

GUÍAS PARA INVESTIGADORES Rev. Methodo 2020;5(3):119-124 https://doi.org/10.22529/me.2020.5(3)09

Recibido 01 Abr. |Publicado 03 Jul. 2020

\title{
¿Dónde publicar?
}

\section{Where to publish a scientific publication?}

\author{
Sandra Gisela Martín ${ }^{1}$ \\ 1Universidad Católica de Córdoba. Directora del Sistema de Bibliotecas. \\ Correspondencia: Sandra G. Martín. Directora Sistema de Bibliotecas. Universidad Católica de Córdoba. Email: dir.biblio@ucc.edu.ar
}

\section{Resumen}

Esta revisión permite conocer las diferentes instancias y criterios para la publicación científica. Otorga al investigador herramientas para la evaluación de cómo y dónde publicar su investigación con el objetivo de que tenga el mayor impacto posible y sea de utilidad para la comunidad científica. Las preguntas que intenta responder son: ¿Dónde público un artículo científico? ¿Qué significa que la revista esté indizada/indexada? ¿Cómo elijo una revista de calidad? ¿Cuáles son las principales bases de datos disponibles?

Palabras clave: Indexación - Base de datos - Publicación científica

\section{Abstract}

This review allows knowing the different instances and criteria for scientific publication. It provides the researcher with tools for evaluating how and where to publish their research so that it has the greatest possible impact and is useful to the scientific community. The questions you are trying to answer are: Where do I publish a scientific article? What does it mean that the magazine is indexed / indexed? How do I choose a quality magazine? What are the main databases available?

Keywords: Indexing - Database - scientific publication

\section{1- ¿Dónde público un artículo científico?}

Day $(2005)^{1}$ define al artículo científico como "un informe escrito y publicado que describe resultados originales de investigación" (p.8). Los artículos científicos deben ser publicados en revistas científicas de calidad y que estén indexadas. Según el glosario Latindex ${ }^{2}$, las revistas de investigación científica son aquellas que 2 publican predominantemente artículos con los resultados de una investigación (provenientes de proyectos de investigación científica financiados con fondos públicos o privados) o estudios originales que proporcionan un aporte a la disciplina de la revista. Se les exige sistema de arbitraje para la aprobación de los artículos.

\section{2- ¿Qué significa que la revista esté indizada/indexada?}

Cuando son incluidas en bases de datos especializadas o multidisciplinares, en texto completo o referenciales de carácter regional y/o internacional, con el objetivo de lograr una mayor visibilidad.

Una revista puede estar indexada en buscadores, redes sociales académicas, directorios, repertorios,

Revista Methodo: Investigación Aplicada a las Ciencias Biológicas. Universidad Católica de Córdoba. Jacinto Ríos 571 Bo Gral. Paz. X5004FXS. Córdoba. Argentina. Tel.: (54) 3514517299 / Correo: methodo@ucc.edu.ar / Web: methodo.ucc.edu.ar | GUÍAS PARA INVESTIGADORES Rev. Methodo 2020;5(3):119-124. 
bases de datos, índices de citas, etc., pero hay que tener en cuenta que existen distintos niveles de recursos y no todos contienen criterios con indicadores de evaluación de la calidad.

\section{3- ¿Cómo elijo una revista de calidad?}

En primer lugar, es importante tomar como referencia las Bases para la Categorización de Publicaciones Periódicas en Ciencias Sociales y Humanidades del CONICET $^{3}$ las 3 cuales establecen 3 niveles de jerarquización de índices y portales bibliográficos científicos:

Nivel 1: Internacional Global y Regional: (Web of Science) ISI, Scopus, ERIH y

demás índices nacionales de excelencia, SciELO.org, CIRC A.

Nivel 2: Internacional y Regional: Se consideran en este grupo las publicaciones con referato no incluidas en el nivel anterior: Editoriales tales como: Sage, Springer, Taylor \& Francis, WILEY, y bases bibliográficas como Francis, Pascal, JSTOR, Redalyc y Núcleo Básico de Revistas Científicas Argentinas.

Nivel 3: Bases Referenciales Bibliográficas y Catálogos Regionales. Se consideran en este grupo las publicaciones con referato no incluidas en los niveles anteriores, tales como: Philosopher's Index, Sociological Abstracts, Anthro ological Literature, ATLA Religion Database,
Communications Abs. Econ Lit Educational Research Abs. (ERA), ERIC; GEOBASE, Historical Abs., Library \& lnformation Science ABS LISA), Linguistics y Language Behaviour Abs. (LLBA), Modem Language Assoc. Abs. (MLA), PAIS Intemational, PSICODOC; PSylnfo, Sociological Abs. Biblioteca Kttual CLACSO, Portal BID, Index Copernicus y Latindex nivel Catálogo.

También podemos clasificar los portales bibliográficos de acuerdo al ámbito geográfico de cobertura de las revistas:

\section{Nivel internacional \\ - Web of Science \\ - Scopus \\ - Scimago SJR \\ - DOAJ \\ - MIAR \\ - ERIH Plus \\ - CIRC \\ - CARHUS Plus}

\author{
Nivel latinoamericano \\ - Latindex Catálogo \\ - RedALyC \\ - Scielo \\ - LILACS \\ - REDIB \\ - Biblat
}

Argentina

- NB Núcleo Básico de Revistas Científicas Argentinas

- Scielo Argentina.

A continuación, se presentan algunos datos básicos de los principales índices que constituyen una referencia para identificar revistas científicas para publicar un artículo:

\begin{tabular}{|c|c|c|c|}
\hline Base de datos & Empresa & Descripción & $\begin{array}{l}\text { Criterios de } \\
\text { inclusión/ } \\
\text { Acceso al listado } \\
\text { de revistas }\end{array}$ \\
\hline WEB OF SCENCE & - Clarivate & $\begin{array}{l}\text { Plataforma que contiene varias } \\
\text { bases de datos con } \\
\text { herramientas de análisis para } \\
\text { valorar la calidad científica a } \\
\text { partir de las citaciones. } \\
\text { https://clarivate.com/webofscie } \\
\text { ncegroup/solutions/web-of-scie } \\
\text { ncel } \\
\text { Nota: el acceso es por pago. } \\
\text { Ninguna biblioteca en } \\
\text { Argentina tiene subscripción. }\end{array}$ & $\begin{array}{l}\text { Journal evaluation } \\
\text { Master Journal List }\end{array}$ \\
\hline Scopus" & $\frac{\text { Finger }}{\text { EISEVIER }}$ & $\begin{array}{l}\text { Base de datos bibliografica de } \\
\text { resúmenes y citas de artículos } \\
\text { de revistas científicas. } \\
\text { https://www.scopus.com/ } \\
\text { Nota: la biblioteca tiene } \\
\text { acceso a Scopus a través de } \\
\text { la Biblioteca Electrónica del } \\
\text { Mincyt (consulte al } \\
\text { bibliotecario). }\end{array}$ & $\begin{array}{l}\text { Criterios de inclusión } \\
\text { Scopus Sources }\end{array}$ \\
\hline
\end{tabular}

Revista Methodo: Investigación Aplicada a las Ciencias Biológicas. Universidad Católica de Córdoba. Jacinto Ríos 571 Bo Gral. Paz. X5004FXS. Córdoba. Argentina. Tel.: (54) 3514517299 / Correo: methodo@ucc.edu.ar / Web: methodo.ucc.edu.ar | GUIAS PARA INVESTIGADORES Rev. Methodo 2020;5(3):119-124. 


\begin{tabular}{|c|c|c|c|}
\hline 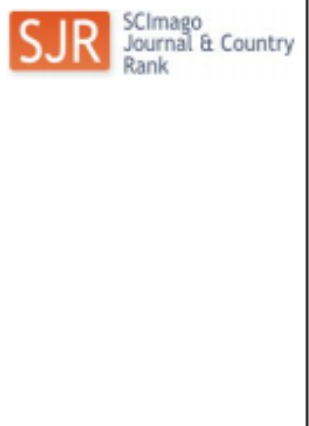 & $\begin{array}{l}\text { Grupo de } \\
\text { investigación } \\
\text { CSIC, } \\
\text { Universidad de } \\
\text { Granada, } \\
\text { Extremadura, } \\
\text { Carlos III } \\
\text { (Madrid) y } \\
\text { Alcalá de } \\
\text { Henares }\end{array}$ & $\begin{array}{l}\text { Portal público que incluye } \\
\text { indicadores cientificos de } \\
\text { revistas y países } \\
\text { desarrollados a partir de la } \\
\text { base de datos Scopus. } \\
\text { https://www.scimagoir.com/ }\end{array}$ & $\begin{array}{l}\text { Criterios de inclusión: } \\
\text { SCOPUS } \\
\text { Journal Ranking }\end{array}$ \\
\hline & $\begin{array}{l}\text { DOAJ, } \\
\text { organización } \\
\text { sin fines de } \\
\text { lucro. }\end{array}$ & $\begin{array}{l}\text { Directorio de revistas } \\
\text { científicas y académicas de } \\
\text { acceso abierto de todo el } \\
\text { mundo. } \\
\text { https://doai.org/ }\end{array}$ & $\begin{array}{l}\text { Criterios de inclusión } \\
\text { Buscar }\end{array}$ \\
\hline 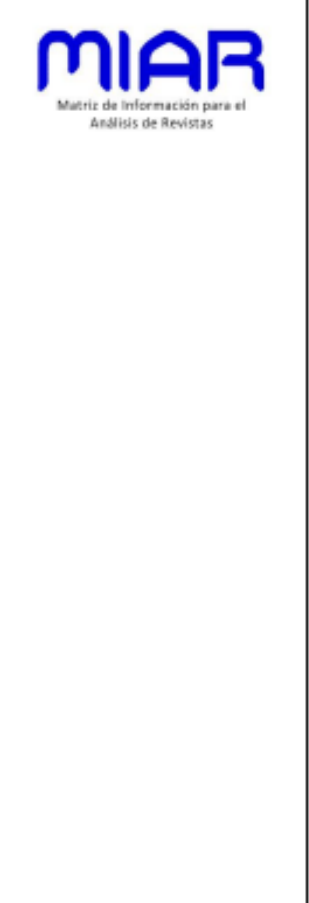 & $\begin{array}{l}\text { Universidad de } \\
\text { Barcelona }\end{array}$ & $\begin{array}{l}\text { Reúne información clave para } \\
\text { la identificación y el análisis de } \\
\text { revistas. Localiza las bases de } \\
\text { datos en que está indizada la } \\
\text { revista y calculará su ICDS } \\
\text { ICDS (Índice Compuesto de } \\
\text { Difusión Secundaria) que es un } \\
\text { indicador que muestra la } \\
\text { visibilidad de la revista en } \\
\text { diferentes bases de datos } \\
\text { cientíicas de alcance } \\
\text { internacional, o en su defecto } \\
\text { en repertorios de evaluación } \\
\text { de publicaciones periódicas. } \\
\text { MIAR ofrece información de la } \\
\text { presencia de la revista en } \\
\text { repertorios de evaluación como } \\
\text { por ejemplo el Sello de Calidad } \\
\text { FECYT, Scimago Journal } \\
\text { Rank, o CARHUS+, } \\
\text { http://miar.ub.edu/ }\end{array}$ & $\begin{array}{l}\text { Criterios de inclusión } \\
\text { Buscar }\end{array}$ \\
\hline 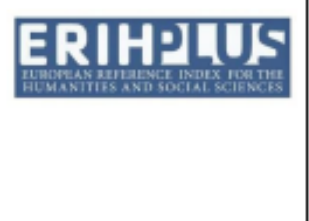 & $\begin{array}{l}\text { Norwegian } \\
\text { Centre for } \\
\text { Research Data } \\
\text { (NSD) }\end{array}$ & $\begin{array}{l}\text { Índice de revistas académico } \\
\text { cientificas en ciencias sociales } \\
\text { y humanidades. } \\
\text { https://dbh.nsd.uib.no/publiseri } \\
\text { ngskanaler/erihplus/ }\end{array}$ & $\begin{array}{l}\text { Criterios de inclusión } \\
\text { Approved journals }\end{array}$ \\
\hline
\end{tabular}

Revista Methodo: Investigación Aplicada a las Ciencias Biológicas. Universidad Católica de Córdoba. Jacinto Ríos 571 Bo Gral. Paz. X5004FXS. Córdoba. Argentina. Tel.: (54) 3514517299 / Correo: methodo@ucc.edu.ar / Web: methodo.ucc.edu.ar | GUIAS PARA INVESTIGADORES Rev. Methodo 2020;5(3):119-124. 


\begin{tabular}{|c|c|c|c|}
\hline & $\begin{array}{l}\text { EC3METRICS } \\
\text { S.L. }\end{array}$ & $\begin{array}{l}\text { Clasificación integrada de } \\
\text { revistas científicas en ciencias } \\
\text { sociales y humanas en función } \\
\text { de su calidad que integra los } \\
\text { productos de evaluación } \\
\text { existentes. } \\
\text { https://www.clasificacioncirc.es } \\
\end{array}$ & $\begin{array}{l}\text { Criterios de inclusión } \\
\text { Buscar }\end{array}$ \\
\hline CARHUS PIUS: & $\begin{array}{l}\text { AGAUR, a } \\
\text { propuesta de } \\
\text { la Direcció } \\
\text { General de } \\
\text { Recerca de la } \\
\text { Generalitat de } \\
\text { Cataluña }\end{array}$ & $\begin{array}{l}\text { Sistema de clasificación de } \\
\text { revistas científicas de los } \\
\text { ámbitos de las ciencias } \\
\text { sociales y humanidades. } \\
\text { http://aqaur.gencat.cat/es/avalu } \\
\text { acio/carhus/l }\end{array}$ & $\begin{array}{l}\text { Criterios de inclusión } \\
\text { Buscador }\end{array}$ \\
\hline & $\begin{array}{l}\text { Universidad } \\
\text { Nacional } \\
\text { Autónoma de } \\
\text { México } \\
\text { (UNAM) }\end{array}$ & $\begin{array}{l}\text { Sistema Regional de } \\
\text { Información en Línea para } \\
\text { Revistas Científicas de } \\
\text { América Latina, el Caribe, } \\
\text { España y Portugal. } \\
\text { https://www.latindex.org/ }\end{array}$ & $\begin{array}{l}\text { Criterios de inclusión } \\
\text { Buscar }\end{array}$ \\
\hline 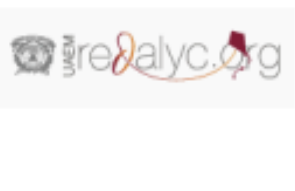 & $\begin{array}{l}\text { Universidad } \\
\text { Autónoma del } \\
\text { Estado de } \\
\text { México }\end{array}$ & $\begin{array}{l}\text { Red de Revistas Cientificas de } \\
\text { América Latina y el Caribe, } \\
\text { España y Portugal. } \\
\text { httos://www redalyc.org/ }\end{array}$ & $\begin{array}{l}\frac{\text { Criterios de }}{\text { evaluación }} \\
\text { Colección de revistas }\end{array}$ \\
\hline 任工O & $\begin{array}{l}\text { FAPESP - } \\
\text { CAPES- } \\
\text { BIREME - } \\
\text { OPS - OMS }\end{array}$ & $\begin{array}{l}\text { Portal de revistas cientificas de } \\
\text { países en desarrollo, } \\
\text { especialmente de América } \\
\text { Latina y el Caribe. } \\
\text { https://scielo.org/es/ }\end{array}$ & $\begin{array}{l}\text { Criterios de inclusión } \\
\text { Revistas }\end{array}$ \\
\hline LILACS & OPS/OMS & $\begin{array}{l}\text { Contiene de artículos de } \\
\text { revistas con peer review, tesis } \\
\text { y disertaciones, documentos } \\
\text { gubernamentales, anales de } \\
\text { congresos y libros, publicados } \\
\text { a partir de } 1982 . \\
\text { Incluye además, revisiones } \\
\text { sistemáticas, ensayos clínicos } \\
\text { controlados aleatorios, síntesis } \\
\text { de evidencias, estudios de } \\
\text { evaluación de tecnologías } \\
\text { sanitarias, estudios de } \\
\text { evaluación económica, guías } \\
\text { de práctica clínica, informes }\end{array}$ & $\begin{array}{l}\text { Criterios de inclusión } \\
\text { Periódicos indexados } \\
\text { por país }\end{array}$ \\
\hline
\end{tabular}

Revista Methodo: Investigación Aplicada a las Ciencias Biológicas. Universidad Católica de Córdoba. Jacinto Ríos 571 Bo Gral. Paz. X5004FXS. Córdoba. Argentina. Tel.: (54) 3514517299 / Correo: methodo@ucc.edu.ar / Web: methodo.ucc.edu.ar | GUIAS PARA INVESTIGADORES Rev. Methodo 2020;5(3):119-124. 


\begin{tabular}{|c|c|c|c|}
\hline & & $\begin{array}{l}\text { técnicos, informes de casos y } \\
\text { otros. } \\
\text { https://lilacs.bvsalud.org/es/ }\end{array}$ & \\
\hline REDIB |Rad berramericana & $\begin{array}{l}\text { CSIC - } \\
\text { Universia } \\
\$ \text { Clarivate } \\
\text { Analytics }\end{array}$ & $\begin{array}{l}\text { Red Iberoamericana de } \\
\text { Innovación y Conocimiento } \\
\text { Cientifico } \\
\text { https://redib.orq/ }\end{array}$ & $\begin{array}{l}\text { Criterios de inclusión } \\
\text { Listado de revistas }\end{array}$ \\
\hline & $\begin{array}{l}\text { Universidad } \\
\text { Nacional } \\
\text { Autónoma de } \\
\text { México } \\
\text { (UNAM) }\end{array}$ & $\begin{array}{l}\text { Bibliografía latinoamericana de } \\
\text { revistas de investigación } \\
\text { cientifica y social. } \\
\text { https://biblat.unam.mx/es/ }\end{array}$ & $\begin{array}{l}\text { Criterios de inclusión } \\
\text { Buscar }\end{array}$ \\
\hline & CONICET & $\begin{array}{l}\text { Determina un conjunto de } \\
\text { publicaciones científicas y } \\
\text { tecnológicas argentinas de } \\
\text { excelencia, en los distintos } \\
\text { campos del conocimiento. } \\
\text { http://www.caicyt-conicet.gov.a } \\
\text { r/sitio/comunicacion-cientifica/n } \\
\text { ucleo-basico/ }\end{array}$ & $\begin{array}{l}\text { Criterios de inclusión } \\
\text { Revistas integrantes }\end{array}$ \\
\hline
\end{tabular}

Para las áreas de ciencias de la salud el índice de referencia por excelencia es MEDLINE.

\begin{tabular}{|l|l|l|l|}
\hline PublMed & $\begin{array}{l}\text { National } \\
\text { Library of } \\
\text { Medicine, } \\
\text { USA }\end{array}$ & $\begin{array}{l}\text { Motor de búsqueda de libre } \\
\text { acceso a la base de datos } \\
\text { MEDLINE de citaciones y } \\
\text { resúmenes de artículos de } \\
\text { investigación biomédica. }\end{array}$ & $\begin{array}{l}\underline{\text { Criterios de }} \\
\text { inclusión } \\
\text { Revistas } \\
\text { indexadas }\end{array}$ \\
\hline
\end{tabular}

\section{4- A tener en cuenta: ¡MUY IMPORTANTE!}

Si la revista está indexada en Google Académico (GS), no implica que sea una revista de calidad. GS incluye todo tipo de recursos sin criterios de selección.

- Si una revista está incluida en Dialnet, no significa que haya sido evaluada con criterios de calidad. Dialnet no es un sistema de evaluación de revistas.
- Si la revista está incluida en alguno de los índices falsos que se mencionan en esta lista https://predatoryjournals.com/metrics/ no es aconsejable seleccionarla para publicar.

- No confundir visibilidad con calidad. Una revista puede ser muy visible, es decir estar incorporada en múltiples bases de datos, pero puede no ser de calidad al no estar incluida en alguno de los repertorios mencionados en el cuadro anterior.

\section{Referencias}

1. Day, R.A. (2005). Cómo escribir y publicar trabajos científicos. 3a. ed. Washington, DC: OPS. (Publicación Científica y Técnica No. 598).

http://www.bvs.hn/Honduras/pdf/Comoescribirypublicar.pdf. 
2. Glosario Latindex https://www.latindex.org/lat/documentos/Glosario_Latindex_esp.pdf.

3. [1] CONICET Resolución 2249/2014. https://www.conicet.gov.ar/bases-para-la-categorizacion-depublicaciones-periodicas-en-ciencias-sociales-y-humanidades/

Biblio 5
GUÍAS

\section{(c) (1)(3)}

Revista Methodo: Investigación Aplicada a las Ciencias Biológicas. Universidad Católica de Córdoba. Jacinto 